\title{
A prospective cohort study on posttraumatic stress disorder in liver transplantation recipients before and after transplantation: Prevalence, symptom occurrence, and intrusive memories
}

\author{
Coby Annema ${ }^{\mathrm{a}, *}$, Gerda Drent ${ }^{\mathrm{b}}$, Petrie F. Roodbol ${ }^{\mathrm{c}}$, Herold J. Metselaar ${ }^{\mathrm{d}}$, Bart Van Hoek ${ }^{\mathrm{e}}$, Robert J. Porte ${ }^{\mathrm{f}}$, \\ Maya J. Schroevers ${ }^{\mathrm{c}}$, Adelita V. Ranchor ${ }^{\mathrm{c}}$ \\ a University of Groningen, University Medical Center Groningen, School of Nursing \& Health, Groningen, The Netherlands \\ ${ }^{\mathrm{b}}$ University of Groningen, University Medical Center Groningen, Department of Gastroenterology and Hepatology, Groningen, The Netherlands \\ c University of Groningen, University Medical Center Groningen, Department of Health Psychology, Groningen, The Netherlands \\ ' Erasmus Medical Center, Department of Gastroenterology and Hepatology, Rotterdam, The Netherlands \\ e Leiden University Medical Center, Department of Gastroenterology and Hepatology, Leiden, The Netherlands \\ ${ }^{\mathrm{f}}$ University of Groningen, University Medical Center Groningen, Department of Surgery, Section of Hepato-Pancreato-Biliary Surgery and Liver Transplantation, Groningen, The Netherlands
}

\section{A R T I C L E I N F O}

\section{Article history:}

Received 3 October 2016

Received in revised form 26 January 2017

Accepted 29 January 2017

\section{Keywords:}

Liver transplantation

Psychological distress

PTSD

Intrusive memories

Transplant candidates

Transplant recipients

\begin{abstract}
A B S T R A C T
Objective: This study aimed at increasing the understanding of posttraumatic stress disorder (PTSD) in liver transplant patients by describing the course of PTSD, symptom occurrence, psychological co-morbidity, and the nature of re-experiencing symptoms.

Methods: A prospective cohort study was performed among 95 liver transplant recipients from before transplantation up until one year post-transplantation. Respondents filled out a questionnaire regarding psychological functioning (PTSD, anxiety, and depression) before, and at 3, 6, and 12 months post-transplantation. Both quantitative and qualitative methods were used to analyze the data.

Results: Before transplantation, respectively $10.5 \%$ and $6.3 \%$ of the respondents were identified as possible cases of full or partial PTSD. In all cases, co-morbid conditions of anxiety and/or depression were present. After transplantation, no new onset of full PTSD was found. New onset of possible partial PTSD was found in six respondents. Arousal symptoms were the most frequently reported symptoms, but may not be distinctive for PTSD in transplant patients because of the overlap with disease- and treatment-related symptoms. Re-experiencing symptoms before transplantation were mostly related to waiting for a donor organ and the upcoming surgery; after transplantation this was related to aspects of the hospital stay.

Conclusions: In our group of liver transplant patients, PTSD symptomatology was more present before transplantation than after transplantation. Being diagnosed with a life-threatening disease seemed to be the main stressor. However, when a diagnosis of PTSD is suspected, assessment by a clinician is warranted because of the overlap with mood and anxiety disorders, and disease- and treatment-related symptoms.
\end{abstract}

(c) 2017 Elsevier Inc. All rights reserved.

\section{Introduction}

Since the introduction of the fourth edition of the Diagnostic and Statistical Manual of Mental Disorders (DSM-IV) [1] in 1994, being diagnosed with a life-threatening illness has been introduced as a potential stressor event for PTSD. Since then, PTSD has been described in a variety of somatic diseases and treatments, including organ transplantation [2-7]. A recent systematic review [8] showed that $0-46 \%$ of transplant recipients had clinically relevant symptom levels of PTSD, while clinician-ascertained PTSD was present in 1-16\% of the cases.

\footnotetext{
* Corresponding author at: School of Nursing \& Health, PO Box 30.001 (FC14), 9700 RB Groningen, The Netherlands.

E-mail address: j.h.annema@umcg.nl (C. Annema).
}

Studies on transplant candidates are limited and mainly retrospective in nature, showing that clinically relevant symptom levels of PTSD were present in $7-25 \%[9,10]$, while $2-6 \%$ of transplant candidates satisfied the criteria for PTSD [11,12].

So far, the focus of the studies on PTSD after organ transplantation has mainly been on assessing prevalence rates, identifying risk factors, and the impact on outcomes after transplantation. Little attention has been paid to which aspects of the transplant process are traumatic in nature, to the occurrence of specific symptoms of PTSD, and to the overlap of PTSD symptoms with disease symptoms and other psychological disorders. Besides this, prospective studies examining the course of PTSD in the same patient group before and after transplantation are lacking. Examining these aspects may help to gain a better understanding of the concept of PTSD in the transplant population. 
PTSD is characterized by symptoms of re-experiencing, avoidance, and arousal [1]. Symptoms of re-experiencing, such as recurrent dreams, intrusive memories, or flashbacks related to the event, are seen as the core symptom of PTSD [13]. However, contrary to other stressful events that may lead to PTSD, such as rape or car accidents, being diagnosed with a life-threatening illness is not a single event but a process, comprising a number of stressors that may lead to a traumatic experience. In the liver transplant process these are, among others, being diagnosed with a potentially life-ending disease, for which a donor organ is needed to survive, but where it is uncertain if this donor organ will arrive in time. Each year approximately $15 \%$ of transplant candidates die while on the organ transplant waiting list [14]. When a donor organ becomes available, patients have to undergo major surgery, followed by a stay on the intensive care unit (ICU) often accompanied by a delirium. After a successful transplantation, patients have to adjust to a life with a life-long regimen of immunosuppressive drugs and life-style rules but they may as well have to deal with serious, potentially life-ending, complications, such as rejection of the graft, or the development of cardiovascular diseases or cancer $[15,16]$. Examining the nature of these symptoms in transplant patients may provide valuable insight into stressors associated with PTSD in this population.

The symptom clusters of avoidance and arousal are more general in nature, and show an overlap with mood and anxiety disorders [17]. Avoidance symptoms refer to the avoidance of distressing thoughts, feelings, or reminders of the event, but also detachment from others, and hopelessness about the future. Arousal symptoms are characterized by aggressive behavior, sleep disorders, and hyper-vigilance.

An important aspect to consider regarding these PTSD symptoms is that they should not be the result of another medical condition, medication, drugs, or alcohol [18]. In liver transplant patients, arousal symptoms like sleeping disorders and concentration problems may also be disease- or treatment-related. Sleeping disorders are common in both transplant candidates (35-73\%) and transplant recipients (41-73\%) mainly due to hepatic encephalopathy, the underlying liver disease or physical problems [19-23]. Concentration problems and irritability may also interfere with symptoms of encephalopathy before transplantation [24]. Therefore, examining the occurrence of PTSD symptoms, in transplant patients can add to the understanding of PTSD in the transplant population.

Because of the overlap of avoidance and arousal symptoms with mood and anxiety disorders, examining comorbidity between PTSD, anxiety, and depression, and the overlap of symptoms of anxiety and depression with the symptom clusters of PTSD may help to differentiate between these problems.

The aim of this study was to increase the understanding of PTSD in liver transplant candidates and recipients by describing the course of PTSD from before transplantation up until the first year after transplantation, symptom occurrence, the overlap of PTSD with anxiety and depression, and to examine the nature of re-experiencing symptoms in liver transplant patients.

\section{Methods}

A prospective cohort study on psychological aspects of liver transplantation was performed among transplant patients in all three liver transplant centers in the Netherlands. Transplant candidates who were placed on the waiting-list between October 2009 and April 2013 were asked to participate. Inclusion criteria were: $\geq 18$ years, and receiving medical treatment in one of the three transplant centers. Exclusion criteria were: unable to fill out a questionnaire due to physical, mental, or cognitive functioning, or due to a language barrier.

Eligible transplant candidates $(n=350)$ received a letter explaining the purpose and procedure of the study, together with an informed consent form and a pre-addressed, stamped return envelope. After written informed consent, respondents received a baseline questionnaire (T0). Measurements of psychological functioning were repeated every six months after inclusion in the study until transplantation. In this study, data from the last measurement-point before the transplant were used to describe PTSD symptoms before transplantation (T0). After transplantation respondents filled out a questionnaire at three (T1), six (T2), and twelve (T3) months after the transplant surgery. The institutional review board of the transplant center that initiated the study approved the study, and a positive recommendation of local feasibility was obtained from the other transplant centers (METc2009.190).

\subsection{Research instruments}

To measure symptoms of PTS, the Self-Rating Inventory for Posttraumatic Stress Disorder (SRIP) was used [25], a Dutch screening instrument that registers symptoms of PTSD. The 22 items, corresponding to the DSM-IV criteria for PTSD, are rated on a 4-point self-report scale ( $1=$ not at all, to $4=$ extremely). The SRIP has satisfying psychometric properties: validity (0.90), reliability (0.92), sensitivity (83\%), and specificity (72\%) [25]. In this study Cronbach's alphas of the SRIP were, respectively, 0.89 (T0), 0.88 (T1), 0.87 (T2), and 0.87 (T3).

The items of the SRIP are stated in general terms, by referring to a stressful experience that happened in the past. In order to be able to examine symptoms of PTSD related to the end-stage organ disease (T0) or to the transplantation (T1-T3), the items were adjusted by replacing "stressful event" with either "my disease" or "my transplantation." Respondents who reported having re-experiencing symptoms, such as intrusive thoughts or recurrent dreams, were asked to briefly describe the nature of these re-experiencing symptoms.

In the SRIP, five of the PTSD symptoms mentioned in the DSM-IV are split into two separate items. For example, "having difficulty falling or staying asleep" is split into two items: "having difficulty falling asleep" and "having difficulty staying asleep." To correspond to the DSM-IV criteria, SRIP items that belong to the same PTSD symptom were merged into one item.

A cut-off score of $\geq 39$ was used to identify respondents with clinically relevant symptom levels of PTS [26]. To be able to identify possible cases of PTSD, all items were recoded into 0 (no symptom of PTSD, scores 1 or 2 ) and 1 (symptom of PTSD, scores 3 or 4 ). For each symptom cluster, the number of symptoms was calculated by adding up the recoded symptom scores. Based on DSM-IV-criteria, possible cases of full PTSD were defined as the presence of one symptom of re-experiencing, three avoidance symptoms, and two arousal symptoms [1]. Regarding possible cases of partial PTSD, different criteria have been used in the literature, either satisfying symptom clusters at two of the three symptom clusters [27], or having one symptom in each symptom cluster [28]. Because intrusive re-experiencing is recognized as the core symptom of PTSD, the latter definition of partial PTSD was used in this study.

To measure psychological co-morbidity, the symptoms of anxiety and depression were assessed using, respectively, the short form of the State-Trait Anxiety Inventory (STAI-6) [29] and the Center for Epidemiological Studies Depression Scale (CES-D) [30].

The STAI- 6 consists of 6 items rated on a 4-point intensity scale $(1=$ not at all, to $4=$ very much), resulting in a total sum score between 6 and 24 . Higher scores indicate more symptoms of anxiety. Based on a transformation of the original 20 item scale cut-off of $\geq 40$ for the general population [31], a cut-off score of $\geq 12$ was used to identify clinically relevant cases. The convergent validity of the STAI- 6 with the full form of the STAI showed a correlation of 0.95 [32]. Cronbach's alpha of the STAI-6 in the present study varied from 0.87 to 0.89 at the different measurement-points.

The CES-D consists of 20 items, scored on a 4-point self-report scale ( $0=$ seldom or never, to $4=$ most of the time-always). Higher scores indicate more symptoms of depression. A cut-off score of $\geq 16$ was used to identify clinically relevant cases [33]. Cronbach's alpha of the CES-D in the present study varied from 0.91 to 0.92 at the different measurement-points. 
Demographic variables regarding age, sex, marital status, educational level, nationality, and employment status were retrieved by self-report. Clinical data regarding primary liver disease and time on waiting-list were obtained from the medical record.

\subsection{Statistical analyses}

Descriptive statistics were used to calculate mean or median scores, and prevalence rates regarding demographic and clinical characteristics, and prevalence and incidence rates of full and partial PTSD. Differences between groups were analyzed using Fisher's exact test or $\chi^{2}$-test for nominal variables. Because of non-normal distribution, the Mann-Whitney $U$ test was used to analyze differences between groups on continuous variables.

Pearson's correlation coefficient was used to examine the association between the symptom clusters of PTSD with symptoms of anxiety and depression.

To analyze the qualitative data regarding re-experiencing symptoms, content analysis was performed by two researchers (GD/CA), independent of each other. Data were examined using a direct approach with a priori categories based on the re-experiencing symptoms in the DSM-IV diagnostic criteria for PTSD. Consensus on the coding was reached if the coding corresponded completely or after discussion about the differences in coding. If no consensus was reached, a third researcher (MJS) was asked to examine the specific data. Data were discussed with all researchers involved to reach final consensus.

\section{Results}

\subsection{Study population}

Of the 350 eligible transplant candidates, 241 (69\%) agreed to participate. Of these, 116 received a transplant within the study period. However, for 21 respondents, datasets were incomplete and therefore excluded from analyses. Reasons for missing data were: deceased $(n=12)$, hospitalization at measurement-point $(n=3)$, lost to follow-up ( $n=3)$, questionnaire not returned $(n=2)$, and re-transplantation $(n=1)$. Demographic and clinical characteristics of the total study population, and included and excluded respondents are shown in Table 1. No significant differences were found between respondents included or excluded from the analyses.

\subsection{Prevalence and incidence rates of PTSD}

Table 2 shows the prevalence rates of PTSD at the different measurement-points. Clinically relevant symptomatology, based on the cut-off score $(\geq 39)$, as well as possible cases of PTSD, based on criteria for full and partial PTSD, were more present before transplantation, when compared to the period after transplantation. The cumulative incidence, the proportion of individuals newly diagnosed with possible full or partial PTSD before and in the first year after transplantation, was $23.2 \%$ (Table 2). After transplantation, no new onset of full PTSD was found, whereas new onset of possible cases of partial PTSD was found in six transplant recipients.

\subsection{Symptom occurrence}

Fig. 1 provides an overview of the percentage of respondents with clinically relevant symptoms (scores 3 or 4 ) of all PTSD symptoms at the four measurement-points. Regarding re-experiencing symptoms, "recurring dreams" and "intrusive memories" were the most frequently reported symptoms at all measurement-points. In the avoidance symptom cluster, "sense of foreshortened future" and "disinterest in activities" were the most reported symptoms before transplantation. After transplantation, the symptom "forgot important aspects" became most prevalent. The most reported symptoms in the arousal cluster were "having difficulty falling or staying asleep" and "problems concentrating."

\subsection{Psychological comorbidity}

Before transplantation, almost all respondents who were identified as possible cases for either full or partial PTSD, also showed clinically relevant symptoms levels of both depression and anxiety (Fig. 2).

To identify which symptom clusters of PTSD showed an overlap with either anxiety or depression, correlations between the number of

Table 1

Demographic and clinical characteristics of the study population.

\begin{tabular}{|c|c|c|c|c|}
\hline & All respondents $n=116$ & Respondents included in analyses $n=95$ & Respondents excluded from analyses $n=21$ & $P$-Value \\
\hline \multicolumn{5}{|l|}{$n(\%)$} \\
\hline Sex: Male & $76(65.5)$ & $63(66.3)$ & $13(61.9)$ & 0.80 \\
\hline $\begin{array}{l}\text { Living situation: With partner } \\
\text { Educational level }\end{array}$ & $89(76.7)$ & $71(74.7)$ & $18(85.7)$ & 0.40 \\
\hline $\begin{array}{l}\text { - Primary } \\
\text { - Secondary } \\
\text { - University } \\
\text { Employment status }\end{array}$ & $\begin{array}{l}21(18.3) \\
54(47.0) \\
40(34.8)\end{array}$ & $\begin{array}{l}15(16.0) \\
43(45.7) \\
36(38.3)\end{array}$ & $\begin{array}{l}6(28.6) \\
11(52.4) \\
4(19.0)\end{array}$ & 0.18 \\
\hline $\begin{array}{l}\text { - Working } \\
\text { - Sick-leave/disabled } \\
\text { - Retired/homemaker/student } \\
\text { Nationality: Dutch } \\
\text { Primary liver disease }\end{array}$ & $\begin{array}{l}32(27.6) \\
59(50.9) \\
25(21.6) \\
109(94.0)\end{array}$ & $\begin{array}{l}28(29.5) \\
48(50.5) \\
19(20.0) \\
91(95.8)\end{array}$ & $\begin{array}{l}4(19.0) \\
11(52.4) \\
6(28.6) \\
18(85.7)\end{array}$ & 0.11 \\
\hline - Biliary cirrhosis & $40(34.5)$ & $36(37.9)$ & $4(19.0)$ & 0.10 \\
\hline - Metabolic disorder & $14(12.1)$ & $11(11.6)$ & $3(14.3)$ & 0.72 \\
\hline - Cirrhosis unknown etiology & $9(7.8)$ & $6(6.3)$ & $3(14.3)$ & 0.21 \\
\hline - Alcoholic cirrhosis & $26(22.4)$ & $22(23.2)$ & $4(19.0)$ & 0.78 \\
\hline - Viral hepatitis & $17(14.7)$ & $11(11.6)$ & $6(28.6)$ & 0.08 \\
\hline $\begin{array}{l}\text { - Other } \\
\text { Mean (SD)/median (range) }\end{array}$ & $9(7.8)$ & $9(9.5)$ & $0(0)$ & 0.36 \\
\hline Age (at time of transplantation) & $50.8(11.4)$ & $50.3(11.3)$ & $53.4(12.2)$ & 0.20 \\
\hline Time on waiting-list (in months) & $9.4(0.2-77.5)$ & $9.5(0.2-77.5)$ & $8.5(1.0-24.2)$ & 0.49 \\
\hline
\end{tabular}


Table 2

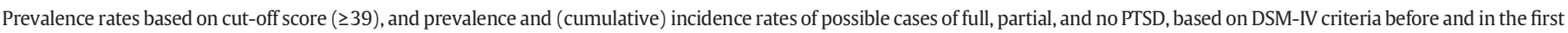
year after transplantation.

\begin{tabular}{|c|c|c|c|c|c|c|c|}
\hline$N=95$ & \multicolumn{2}{|c|}{ Before transplantation } & \multicolumn{2}{|c|}{3 months after transplantation } & \multicolumn{2}{|c|}{6 months after transplantation } & 12 months after transplantation \\
\hline \multicolumn{8}{|c|}{ Point-prevalence (n/\%) } \\
\hline Cut-off $\geq 39$ & $30(31.6)$ & & $15(15.8)$ & & $14(14.7)$ & \multicolumn{2}{|c|}{$14(14.7)$} \\
\hline Full PTSD & $10(10.5)$ & & $1(1.1)$ & & $0(0)$ & \multicolumn{2}{|c|}{$0(0)$} \\
\hline Partial PTSD & $6(6.3)$ & & $5(5.3)$ & & $6(6.3)$ & \multicolumn{2}{|c|}{$3(3.2)$} \\
\hline No PTSD & $79(83.2)$ & & $89(93.6)$ & & $89(93.6)$ & \multicolumn{2}{|c|}{$92(96.8)$} \\
\hline Incidence (n/\%) & Before transplantation & 3 month & fter transplantation & 6 months a & plantation & 12 months after transplantation & Cumulative Incidence (n/\%) \\
\hline Full PTSD & $10(10.5)$ & 0 & & 0 & & 0 & $10(10.5)$ \\
\hline Partial PTSD & $6(6.3)$ & $1(1.1)$ & & $3(3.2)$ & & $2(2.1)$ & $12(12.7)$ \\
\hline Total & $16(16.8)$ & $1(1.1)$ & & $3(3.2)$ & & $2(2.1)$ & $22(23.2)$ \\
\hline
\end{tabular}

symptoms in each cluster with the total score on the STAI and the CES-D were examined. Because of the few possible cases of full and partial PTSD in the post-transplant period, we were only able to perform these analyses using data from the pre-transplant measurement. Regarding anxiety, all symptom clusters of PTSD were significantly correlated with the STAI- 6 total score. The strength of the correlation was moderate for the re-experiencing cluster $(r=0.32)$, and large for both the avoidance symptom cluster $(r=0.55)$ and the arousal symptom cluster $(r=0.57)$. Regarding depression, all symptom clusters of PTSD were also significantly correlated with the CES-D total score. The strength of these correlations was moderate for the re-experiencing cluster $(r=0.39)$, and large for both the avoidance symptom cluster $(r=0.62)$ and the arousal symptom cluster $(r=0.61)$.

\subsection{Nature of re-experiencing symptoms}

Because re-experiencing symptoms are seen as the core symptom of PTSD, we were interested in the nature of these symptoms. Of the 95 respondents, 49 (52\%) described the content of their re-experiencing symptoms at one or more of the measurement-points. Some respondents mentioned the same symptoms at several measurement-points. Symptoms were therefore merged at the individual level at two measurement-points in time: before and after transplantation.

\subsubsection{Symptoms of re-experiencing before transplantation}

Before transplantation, intrusive thoughts related to the transplant were reported by fourteen (15\%) of the respondents. These thoughts were mainly related to the period of waiting for an organ, such as concerns about timely availability of an organ and waiting for "the call." One respondent experienced a failed attempt to transplant, prior to a successful transplant, because the donor organ was rejected at the final decision, leaving the respondent with concerns about the success of any upcoming organ offer. Other respondents reported that they worried about the transplantation itself. They were concerned about being physically unable to undergo a transplantation because of their deteriorating health status or about the success of the transplant surgery. In addition, concerns about their family were described, mostly in terms of leaving their loved ones behind in case the transplant would not be in time or would be unsuccessful.

Two respondents mentioned that they had recurrent dreams about the transplantation or about death. Three respondents mentioned that they felt distress from cues related to medical complications or to the death of a family member with the same liver disease, leaving them with feelings of anxiety for their own future.

\subsubsection{Symptoms of re-experiencing after transplantation}

After transplantation, 32 (34\%) of the respondents reported one or more re-experiencing symptoms. Twenty-one respondents reported having intrusive memories or thoughts about the transplant, mostly related to the clinical phase after the transplantation, such as the stay on the ICU or the nursing ward, but also regarding specific aspects of the clinical phase, such as experiencing delirium, interventions that restricted freedom of movement, or the feeling of being totally dependent upon others. Besides this, fears concerning the future, for example about the physical recovery or the possibility of graft loss, were described. Intrusive thoughts related to the death of the donor were also reported.

Recurrent dreams or nightmares about the transplantation were reported by fourteen respondents. These dreams were mostly about aspects of the transplant process, such as the surgery or the ICU stay, but unrealistic dreams were also present (e.g., being hunted by sharks, horror-like dreams). One respondent described a feeling of reliving that consisted of a sensation of choking, which reminded of an experience during the stay on the ICU. Distress at cues was mentioned by eight respondents. These cues were related to medical complications, such as recurrence of liver disease or signs of rejection, but also sounds or situations that reminded them of the hospital stay. Only one respondent reported physiological reactions to cues. This respondent felt nausea when confronted with reminders of the hospital stay.

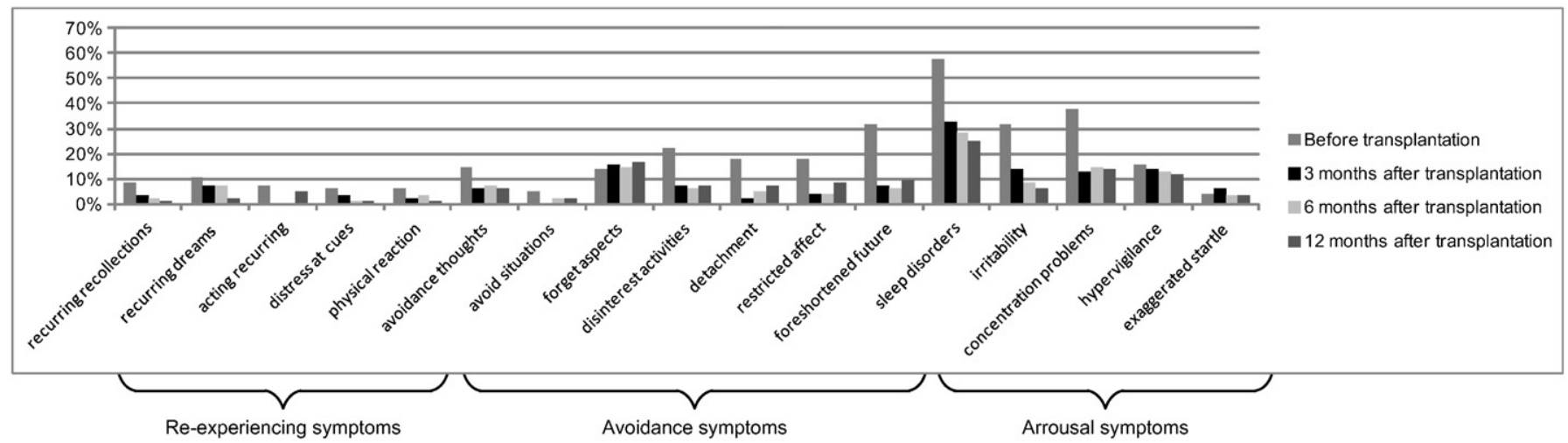

Fig. 1. overview of respondents (\%) with specific PTSD symptoms at the different measurement-points. 


\section{Before transplantation $(n=16)$}

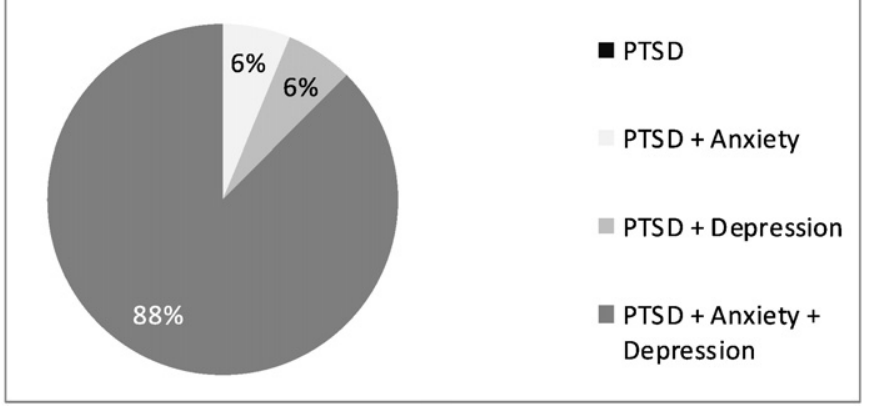

Fig. 2. Percentage of respondents with possible cases of full or partial PTSD and comorbidity of anxiety and/or depression before transplantation.

\section{Discussion}

The results of this study showed that in our population of liver transplant patients clinically relevant PTS symptomatology was more present than possible cases of either full or partial PTSD, and that both PTSD symptomatology and possible cases were more prevalent before than in the first year after transplantation. Remarkably, we found no new onset of full PTSD and only a few possible cases of new onset of partial PTSD after transplantation. All respondents who were identified as possible cases of either partial or full PTSD before transplantation also showed clinically relevant symptom levels of anxiety and/or depression.

Regarding symptom occurrence, arousal symptoms were most present at all measurement-points, especially sleeping disorders and concentration problems.

Our qualitative data showed that in our study population symptoms of re-experiencing before transplantation were mainly related to the wait for a suitable donor and the upcoming transplant surgery; after transplantation mainly to the clinical phase after the transplant surgery and concerns about the future.

Our findings regarding prevalence rates are in line with other studies that show that PTSD symptomatology is higher than possible cases of PTSD in patients after medical illness and treatment [34], and after liver transplantation $[27,35]$. However, we found more possible cases of PTSD before the transplantation than previous studies among transplant candidates $[9,11,12]$.

Based on our qualitative data, the most prominent stressor for the development of PTSD in our group of Dutch liver transplant patients seemed to be "being diagnosed with a life-threatening disease." The nature of the re-experiencing symptoms, as described by the respondents, showed that, before transplantation, the unpredictability of the timing of the transplantation, along with deterioration in health status, left transplant candidates not only with concerns about the timely availability of a donor organ but also with concerns about leaving their loved ones behind.

Symptoms of re-experiencing after the transplantation were mostly related to the clinical phase after the transplantation (e.g., ICU stay, delirium) but also represented current stressors like concerns about the recovery, or conceived future events like fear of graft loss. Although $34 \%$ of the respondents reported having intrusive thoughts, dreams, or distress at cues after the transplantation, this did not lead to the new onset of full PTSD; furthermore, only 6\% of the respondents were identified as possible new cases of partial PTSD. This might indicate that, after a successful transplantation, most of the respondents were capable of successfully processing their transplant experience. In earlier studies a stay on the ICU and delirium have been identified as potential risk factors for PTSD [36-39]. However, the results of our study do not support this finding.
Although arousal symptoms were most present at all measurementpoints, the presence of arousal symptoms may not be indicative of PTSD in the liver transplant population because of the overlap with disease and treatment-related problems and other psychological disorders. Sleeping disorders and concentration problems are common in transplant patients, mainly due to physical problems [19-23]. Therefore, when transplant patients report arousal symptoms, causes other than PTSD should be kept in mind. Moreover, we found that PTSD symptomatology in liver transplant patients was often accompanied by co-morbid conditions of anxiety and/or depression, and that, especially, avoidance and arousal symptoms showed strong correlations with high symptom levels of anxiety and depression.

Due to the overlap between symptoms of PTSD with disease and treatment-related symptoms, and with other psychological disorders, the presence of PTSD in the transplant population could easily be overestimated. Because it is difficult to disentangle differences between them, a structured or semi-structured diagnostic interview by a clinician is warranted to confirm the diagnosis when PTSD is suspected. In this assessment, anxiety and depression should also be considered. Furthermore, alternative diagnoses, such as an acute stress disorder should be hold in mind, because some of the re-experiencing symptoms described by the respondents were related to current or conceived events (medical complications, fear of graft failure), which could be indicative of an acute stress disorder.

\subsection{Strengths and limitations}

The strength of our study is the prospective, longitudinal design and the satisfactory response rate (69\%) which made it possible to follow the course of PTSD in our patient group over time. To our knowledge, no other studies have investigated PTSD in a transplant population from the before transplantation up until one year after the transplantation. However, the generalizability of our results for the liver transplant population as a whole may be limited. Due to the sample size $(n=95)$ the results need to be interpreted with caution. Besides this, we could not include transplant recipients who were transplanted soon after placement on the waiting list or patients with acute liver failure because of the prospective design. In this specific patient group, the transplantation itself may have a different impact, as shown by Guimaro and colleagues [40], who found high symptom levels of PTS (46\%) in patients transplanted for acute liver failure. Therefore, the course of PTSD in patients with an acute onset of their liver disease or who were on the waiting list for a short period of time, needs to be examined in future research.

Another limitation of our study was that, because the start of the study was before the introduction of the DSM-5 [18], we were not able to examine PTSD in our population based on the latest insights. However, a study by O'Donnell and colleagues [41] showed that the prevalence scoring under DSM-5 was not significantly different from DSM-IV. Therefore, the results of our study may also be representative for DSM-5 criteria.

Also, the use of only self-report to assess symptoms of PTSD can be seen as a limitation. In future research, a clinician-ascertained diagnosis of PTSD may have added value. Also, the nature of the re-experiencing symptoms was only assessed by self-report. As a consequence of this, not all respondents who indicated having intrusive memories or dreams described the content of these thoughts or dreams. In addition, probing questions aimed at gaining more in-depth understanding of the nature of the re-experiencing symptoms was not possible. For future research, we suggest using interviews to obtain a more in-depth understanding of these symptoms.

In conclusion, in our group of Dutch liver transplant patients PTSD symptomatology was more present than possible cases of PTSD. Also, more patients were identified as probable cases of either full or partial PTSD before transplantation - often accompanied by co-morbid conditions of anxiety and/or depression - than after the transplant. Therefore, 
being diagnosed with a life-threatening disease seemed to be the main stressor for PTSD. Although aspects related to the transplantation itself, such as the stay on the ICU or delirium, were described as stressors after the transplant, this did not lead to development of full or partial PTSD after transplantation. Arousal symptoms, such as sleeping disorders and concentration problems, were most frequently reported by transplant patients, but may not be indicative of PTSD because of the overlap with disease and treatment-related factors. Therefore, healthcare workers should especially be aware of the possibility of PTSD when symptoms of re-experiencing are reported by transplant patients. Considering the overlap with disease and treatment-related factors and with other psychological disorders, it is important to refer to a clinician when PTSD is suspected in order to confirm the diagnosis and subsequently initiate appropriate interventions.

\section{Conflict of interest statement}

The authors do not have competing interest to report nor received additional funding.

\section{References}

[1] American Psychiatric Association, Diagnostic and Statistical Manual of Mental Disorders. Washington DC: Am. Psych. Assoc, 1994.

[2] G. Abbey, S.B.N. Thompson, T. Hickish, D. Heathcote, A meta-analysis of prevalence rates and moderating factors for cancer-related post-traumatic stress disorder, Psychooncology 24 (2015) 371-381.

[3] M.R. Widows, P.B. Jacobsen, K.K. Fields, Relation of psychological vulnerability factors to posttraumatic stress disorder symptomatology in bone marrow transplant recipients, Psychosom. Med. 62 (2000) 873-882.

[4] J. Hefner, M. Kapp, K. Drebinger, A. Dannenmann, H. Einsele, G. Grigoleit, et al., High prevalence of distress in patients after allogeneic hematopoietic SCT: fear of progression is associated with a younger age, Bone Marrow Transplant. 49 (2014) 581-584.

[5] K. Ginzburg, T. Ein-Dor, Posttraumatic stress syndromes and health-related quality of life following myocardial infarction: 8-year follow-up, Gen. Hosp. Psychiatry 33 (2011) 565-571 (7p).

[6] J. Radcliffe, C.L. Fleisher, L.A. Hawkins, M. Tanney, N. Kassam-Adams, C. Ambrose, et al., Posttraumatic stress and trauma history in adolescents and young adults with HIV, AIDS Patient Care STDs 21 (2007) 501-508 (8p).

[7] C. Annema, P.F. Roodbol, R.E. Stewart, R.J. Porte, A.V. Ranchor, Prevalence of psychological problems and associated transplant-related variables at different time periods after liver transplantation, Liver Transpl. 21 (2015) 524-538.

[8] D.S. Davydow, E.D. Lease, J.D. Reyes, Posttraumatic stress disorder in organ trans plant recipients: a systematic review, Gen. Hosp. Psychiatry 37 (2015) 387-398.

[9] D.G. Cohen, J.D. Christie, B.J. Anderson, J.M. Diamond, R.P. Judy, R.J. Shah, et al., Cognitive function, mental health, and health-related quality of life after lung transplantation, Ann. Am. Thorac. Soc. 11 (2014) 522-530.

[10] J. Jacobs, T. Michael, S. Brandsch, H. Schäfers, H. Wilkens, V. Köllner, The prevalence of posttraumatic stress disorder in lung transplant candidates and recipients, Psychother. Psychosom. Med. Psychol. 65 (2015) 255-260.

[11] L.D. Evans, E.M. Stock, J.E. Zeber, S.B. Morissette, A.A. MacCarthy, E.Y. Sako, et al. Posttransplantation outcomes in veterans with serious mental illness, Transplantation 99 (2015) e57-e65.

[12] S.S. Rogal, D. Landsittel, O. Surman, R.T. Chung, A. Rutherford, Pretransplant depression, antidepressant use, and outcomes of orthotopic liver transplantation, Liver Transpl. 17 (2011) 251-260.

[13] A. Hackmann, A. Ehlers, A. Speckens, D.M. Clark, Characteristics and content of intrusive memories in PTSD and their changes with treatment, J. Trauma. Stress. 17 (2004) 231-240

[14] Dutch Transplantation Society, Annual Report 2014, Leiden, 2015.

[15] K.B.L. Lim, T.D. Schiano, Long-term outcome after liver transplantation, Mt Sinai J. Med. 79 (2012) 169-189.

[16] L. de Kroon, G. Drent, A.P. van den Berg, E.B. Haagsma, Current health status of patients who have survived for more than 15 years after liver transplantation, Neth. J. Med. 65 (2007) 252-258.
[17] W.V. Vieweg, D.A. Julius, A. Fernandez, M. Beatty-Brooks, J.M. Hettema, A.K. Pandurangi, Posttraumatic stress disorder: clinical features, pathophysiology, and treatment, Am. J. Med. 119 (2006) 383-390.

[18] American Psychiatric Association, Diagnostic and Statistical Manual of Mental Disorders. Washington, DC: APA, 2013.

[19] S. De Cruz, J.R.D. Espiritu, M. Zeidler, T.S. Wang, Sleep disorders in chronic liver disease, Semin. Respir. Crit. Care Med. 33 (2012) 26-35.

[20] J.R. Rodrigue, D.R. Nelson, A.I. Reed, D.W. Hanto, M. Curry, Fatigue and sleep quality before and after liver transplantation, Prog. Transplant. 20 (2010) 221-233.

[21] H. Burkhalter, D.P. Brunner, A. Wirz-Justice, C. Cajochen, T.E. Weaver, J. Steiger, et al., Self-reported sleep disturbances in renal transplant recipients, BMC Nephrol. 14 (2013) 220.

[22] M. Akahoshi, T. Ichikawa, N. Taura, H. Miyaaki, T. Yamaguchi, E. Yoshimura, et al., Sleep disturbances and quality of life in patients after living donor liver transplantation, Transplant. Proc. 46 (2014) 3515-3522.

[23] M. Reilly-Spong, T. Park, C.R. Gross, Poor sleep in organ transplant recipients: self-reports and actigraphy, Clin. Transpl. 27 (2013) 901-913.

[24] V.P. Grover, J.M. Tognarelli, N. Massie, M.M. Crossey, N.A. Cook, S. Taylor-Robinson, The why and wherefore of hepatic encephalopathy, Int. J. Gen. Med. 8 (2015) 381-390.

[25] J.E. Hovens, P.R. Falger, W. Op den Velde, P. Meijer, J.H. de Groen, H. van Duijn, A selfrating scale for the assessment of posttraumatic stress disorder in Dutch Resistance veterans of World War II, J. Clin. Psychol. 49 (1993) 196-203.

[26] W.H. van Zelst, E. de Beurs, A.T. Beekman, D.J. Deeg, I. Bramsen, R. van Dyck, Criterion validity of the self-rating inventory for posttraumatic stress disorder (SRIP) in the community of older adults, J. Affect. Disord. 76 (2003) 229-235.

[27] S.G. Jin, L.N. Yan, B. Xiang, B. Li, T.F. Wen, J.C. Zhao, et al., Posttraumatic stress disorder after liver transplantation, Hepatobiliary Pancreat. Dis. Int. 11 (2012) 28-33.

[28] A. Favaro, G. Gerosa, A.L.P. Caforio, B. Volpe, G. Rupolo, D. Zarneri, et al., Posttraumatic stress disorder and depression in heart transplantation recipients: the relationship with outcome and adherence to medical treatment, Gen. Hosp. Psychiatry 33 (2011) 1-7.

[29] T.M. Marteau, H. Bekker, The development of a six-item short-form of the state scale of the Spielberger state-trait anxiety inventory (STAI), Br. J. Clin. Psychol. 31 (1992) 301-306.

[30] J. Bouma, A.V. Ranchor, R. Sanderman, E. Van Sonderen, Measurement of Depressive Symptoms with the CES-D. A Manual (in Dutch), Groningen, Northern Center of Health Research, 1995.

[31] C.D. Spielberger, R.L. Gorsuch, R. Lushene, P.R. Vagg, G.A. Jacobs, Manual for the State-Trait Anxiety Inventory (Self-evaluation Questionnaire), Consulting Psychologists Press, Palo Alto, CA., 1983

[32] A.K. Van der Bij, S. De Weerd, R.J.L.M. Cikot, E.A.P. Steegers, J.C. Braspenning, Validation of the Dutch short form of the state scale of the Spielberger state-trait anxiety Inventory: considerations for usage in screening outcomes, Community Genet. 6 (2003) 84-87.

[33] L.S. Radloff, The CES-D Scale: a self-report depression scale for research in the general population, Appl. Psychol. Meas. 1 (1977) 385-401.

[34] J.E. Tedstone, N. Tarrier, Posttraumatic stress disorder following medical illness and treatment, Clin. Psychol. Rev. 23 (2003) 409-448.

[35] I. Fukunishi, Y. Sugawara, T. Takayama, M. Makuuchi, H. Kawarasaki, Y. Kita, et al. Psychiatric problems in living-related transplantation (I): incidence rate of psychiatric disorders in living-related transplantation, Transplant. Proc. 34 (2002) 2630-2631.

[36] D.S. Davydow, Douglas Zatzick, C.L. Hough, W.J. Katon, A longitudinal investigation of posttraumatic stress and depressive symptoms over the course of the year following medical-surgical intensive care unit admission, Gen. Hosp. Psychiatry 35 (2013) 226-232 (7p).

[37] J.R. Basinski, C.M. Alfano, W.J. Katon, K.L. Syrjala, J.R. Fann, Impact of delirium on distress, health-related quality of life, and cognition 6 months and 1 year after hematopoietic cell transplant, Biol. Blood Marrow Transplant. 16 (2010) 824-831.

[38] D. Bulic, M. Bennet, Y. Shehabi, Delirium in the intensive care unit and long-term cognitive and psychosocial functioning: literature review, Aust. J. Adv. Nurs. 33 (2015) 44-52 (9p).

[39] I. Corrigan, KA.M. Samuelson, B. Fridlund, B. Thomé The meaning of posttraumatic stress-reactions following critical illness or injury and intensive care treatment, Intensive Crit. Care Nurs. 23 (2007) 206-215 (10p).

[40] M.S. Guimaro, S.S. Lacerda, M.R. Aguilar, C.H. Karam, A.M. Kernkraut, B. Ferraz-Neto, Post-traumatic stress disorders, mood disorders, and quality of life in transplant recipients with acute liver failure, Transplant. Proc. 43 (2011) 187-188.

[41] M.L. O'Donnell, N. Alkemade, A. Nickerson, M. Creamer, McFarlane AC, D. Silove, et al., Impact of the diagnostic changes to post-traumatic stress disorder for DSM5 and the proposed changes to ICD-11, Br. J. Psychiatry 205 (2014) 230-235. 